\title{
Lymphadenectomy along the infrapyloric artery may be dispensable when performing pylorus-preserving gastrectomy for early middle-third gastric cancer
}

\author{
Aya Mizuno $^{1} \cdot$ Hisashi Shinohara ${ }^{1,2}(\mathbb{D}) \cdot$ Shusuke Haruta $^{1} \cdot$ Shigeru Tsunoda $^{2}$ • \\ Yasunori Kurahashi $^{3} \cdot$ Yu Ohkura $^{1} \cdot$ Harushi Udagawa $^{1} \cdot$ Yoshiharu Sakai $^{2}$
}

Received: 12 May 2016/Accepted: 2 August 2016/Published online: 11 August 2016

(c) The International Gastric Cancer Association and The Japanese Gastric Cancer Association 2016

\begin{abstract}
This study investigated the incidence of gastric cancer metastasis to the lymph nodes along the infrapyloric artery (IPA), namely no. 6i, by reviewing our medical records of 348 patients who underwent complete no. 6 dissection. Metastasis to these nodes was observed in 11 $(3.2 \%)$ patients. In these patients, one huge tumor was located in the middle third and ten including two early tumors were located in the lower third; the metastasis rate in early lower-third tumors was $2.1 \%$ and reached $19.5 \%$ in advanced tumors. In contrast, no early middle-third gastric cancers had no. 6i metastasis. The median diameter of 6i-positive tumors was 62 (range 18-115) $\mathrm{mm}$, and the distance from the distal tumor border to the pyloric ring was no more than $44 \mathrm{~mm}$. Lymphadenectomy along the IPA is important for treating gastric cancer invading the antrum, but may be dispensable when performing pyloruspreserving gastrectomy for early middle-third cancer.
\end{abstract}

Keywords Infrapyloric artery - Infrapyloric lymph nodes · Pylorus-preserving gastrectomy · Lymphadenectomy ·

Gastric cancer

Aya Mizuno and Hisashi Shinohara contributed equally to this work.

Hisashi Shinohara

shinosug@kuhp.kyoto-u.ac.jp

1 Department of Gastroenterological Surgery, Toranomon Hospital, Tokyo, Japan

2 Department of Surgery, Kyoto University Graduate School of Medicine, 54 Shogoin Kawara-cho, Sakyo-ku, Kyoto 606-8507, Japan

3 Department of Surgery, Hyogo Prefectural Amagasaki General Medical Center, Hyogo, Japan

\section{Introduction}

The no. 6 infrapyloric lymph nodes (LNs) are important perigastric nodes that drain the lymphatic flow from the greater curvature of the stomach [1,2]. The Japanese Gastric Cancer Association (JGCA) has defined this nodal station as the area including the "afferent" LNs along the proximal part of the right gastroepiploic artery (RGEA) and the "efferent" LNs along the right gastroepiploic vein (RGEV) [3]. This definition is sensible because most lymphatic channels are accompanied by blood vessels. The infrapyloric artery (IPA) is the main nutrient vessel supplying the pylorus, including the area $3-5 \mathrm{~cm}$ proximal to the greater curvature to the duodenal bulb $[4,5]$. Our recent study revealed that the IPA diverges independently with RGEA in $76.9 \%$ patients and is closely associated with a certain number of LNs, namely no. 6i [6]. These LNs may be assigned to other afferent lymphatic channels draining the antrum. However, to date, there is limited knowledge about the frequency of metastases to these LNs in gastric cancer. If metastasis to no. $6 \mathrm{i}$ LNs is associated with cancer located in the antrum only, lymphadenectomy along the IPA does not contribute significantly to a cure when performing pylorus-preserving gastrectomy (PPG). The aim of this study was to analyze the incidence of metastasis at no. $6 \mathrm{i}$ LNs depending on the tumor location and depth of invasion and to discuss the significance of lymphadenectomy in gastric cancer surgery.

\section{Patients and methods}

\section{Patients}

This study included 348 gastric cancer patients who underwent total or distal radical gastrectomy with complete 
no. 6 lymphadenectomy from December 2011 through June 2015 at Toranomon Hospital, Tokyo, Japan. Tumor stage was classified according to the 7th edition of the TNM classification [7], and macroscopic type was classified by the Japanese classification [3].

\section{Preparation for LN examination}

Surgical procedures for no. 6 lymphadenectomy were described in detail elsewhere $[6,8,9]$. After surgery, all regional LNs were individually retrieved from the adipose tissue of the surgical specimen. The no. 6 nodes were divided into three substations as per our previous report: $6 \mathrm{a}$ is adjacent to the RGEA from its first gastric branch to its root; $6 \mathrm{v}$ is adjacent to the RGEV, between the root of the RGEA and the confluence of the anterior superior pancreatoduodenal vein, and $6 \mathrm{i}$ is located along the IPA [6, 8]. Figure 1 shows a representative surgical specimen, demonstrating the infrapyloric lymph station divided into three sections. Section $6 i$ was distinguished from section 6a along the avascular area between the proximal branch of the IPA and the RGEA.

\section{Statistics}

Statistical analysis was performed using the Pearson's chisquared test. A $P$ value $<0.05$ was regarded as statistically significant.

\section{Results}

\section{Patient background}

The background clinicopathological characteristics of 348 patients (234 males and 114 females) are given in

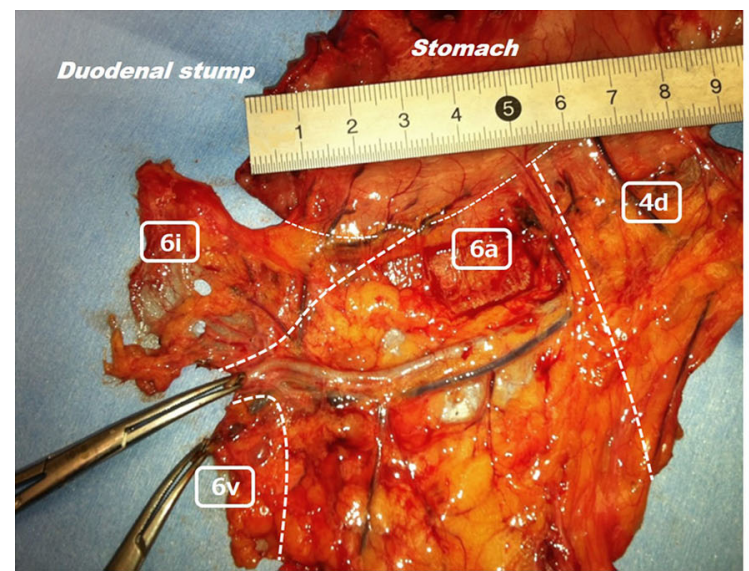

Fig. 1 Representative surgical specimen demonstrating the infrapyloric lymph station divided into three sections. In the right panel, the right gastroepiploic artery (RGEA), right gastroepiploic vein
Table 1 . The most frequent location of the primary tumor was in the middle stomach (M) $(n=164,47.1 \%)$, followed by the lower stomach (L) $(n=136,39.1 \%)$ and upper stomach (U) $(n=48,13.8 \%)$. The proportion of early gastric cancer was $67.8 \%$. Overall, $89(25.6 \%)$ patients had nodal metastasis. Distal gastrectomy and total gastrectomy were performed in 266 and 82 patients, respectively.

\section{Metastasis at no. 6 lymph nodes}

The median numbers of four (interquartile range 3-6), two (1-4), and two (0-3) LNs were retrieved at nos. 6a, $6 \mathrm{v}$, and $6 \mathrm{i}$ substations, respectively. Metastases to no. 6 LNs were observed in $31(8.9 \%)$ patients. The number of patients and metastasis rates at each no. 6 substation according to the tumor location and depth of invasion are summarized in Table 2. No patients with tumors located in $\mathrm{U}$ had nodal metastasis to any no. 6 substations, whereas 31 out of $300(10.3 \%)$ distal two-third gastric cancers had no. 6 metastasis. The rate of metastasis from advanced lower-third tumors of $45.3 \%$ was significantly $(P<0.01)$ higher than that from middle-third tumors $(8.5 \%)$. Number $6 a$ was the most frequent substation for metastasis $(n=19, \quad 5.5 \%)$, followed by no. $6 \mathrm{v}(n=15,4.3 \%)$ and $6 \mathrm{i}(n=11$, $3.2 \%$ ), including ten double metastases (nos. $6 \mathrm{a}+6 \mathrm{v}$ in 5 , nos. $6 \mathrm{a}+6 \mathrm{i}$ in 4 , and nos. $6 \mathrm{v}+6 \mathrm{i}$ in 1 ) and two triple metastases. Metastasis to no. 6i LNs was observed in 11 patients. The majority (10 out of 11 ) of tumors including two early tumors were located in L. The no. $6 \mathrm{i}$ metastasis rates in early and advanced lower-third tumors were 2.1 and $19.5 \%$, respectively. Notably, none of 117 early middle-third gastric cancers had no. $6 \mathrm{i}$ or $6 \mathrm{v}$ metastasis.

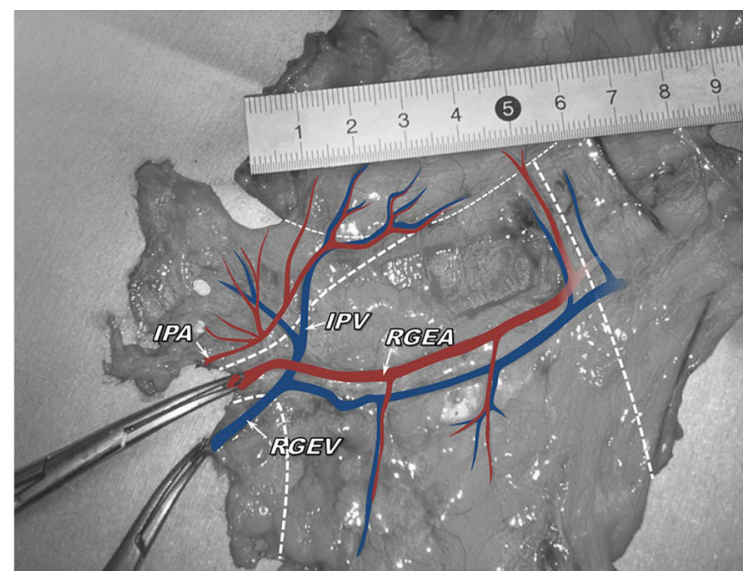

(RGEV), infrapyloric artery (IPA), and infrapyloric vein (IPV) are enhanced by red or blue colors 
Table 1 Characteristics of the 348 patients who underwent gastrectomy

Table 2 Number of patients and metastasis rates at no. 6 lymph node substations according to the location and $\mathrm{T}$ stage

\begin{tabular}{lc}
\hline Variables & $N(\%)$ \\
\hline Sex & \\
Male & $234(67.2)$ \\
Female & $114(32.8)$ \\
Tumor location & \\
U & $48(13.8)$ \\
M & $164(47.1)$ \\
L & $136(39.1)$ \\
Macroscopic types & \\
0 & $241(69.2)$ \\
1 & $10(2.8)$ \\
2 & $29(8.3)$ \\
3 & $59(19.5)$ \\
4 & $9(2.5)$ \\
Histological type & \\
Differentiated & $133(38.2)$ \\
Undifferentiated & $210(60.3)$ \\
Others & $5(1.4)$ \\
Depth of tumor invasion \\
T1 & $236(67.8)$ \\
T2 & $37(10.6)$ \\
T3 & $37(10.6)$ \\
T4 & $38(10.9)$ \\
Nodal involvement & \\
N0 & $259(74.4)$ \\
N1 & $34(9.8)$ \\
N2 & $32(9.2)$ \\
N3 & $23(6.6)$ \\
Tastrectomy & \\
Distal & \\
\hline & \\
& \\
Total & \\
& \\
&
\end{tabular}

\section{Characteristics of no. 6i-positive tumors}

Clinicopathological features of 11 tumors with no. $6 \mathrm{i}$ metastasis are shown in Fig. 2. Except one huge tumor whose diameter was $115 \mathrm{~mm}$ (case 11), ten were located in L. The depth of tumor invasion was defined as T1b in two patients, T3 in two, and T4a in seven. Macroscopic types included two 0 -IIc cases, three type 2, five type 3, and one type 4 cases. The median tumor diameter was 62 (range 18-115) $\mathrm{mm}$, and the median distance from the distal tumor border to the pyloric ring was 14 (range 0-44) $\mathrm{mm}$. The distances in two early tumors (cases 1,2 ) were very close to the pylorus $(20,0 \mathrm{~mm})$. Histological examination did not show any notable characteristics in no. 6i-positive tumors (differentiated type in 4 and undifferentiated type in 7 ); this histological ratio was similar to the overall ratio in 348 cases as shown in Table 1. All advanced tumors had LN metastases to plural stations, although both of the T1 tumors with no. 6i metastasis had no other metastatic LN stations.

\section{Discussion}

The no. 6 infrapyloric LN station is considered an important confluence of lymphatic channels draining the distal two-thirds of the stomach $[1,8]$. The dissection of the no. 6 station is an important surgical step because several studies demonstrated that metastasis to the no. 6 nodes is very common $[2,10]$. Our present results showing that nearly $10 \%$ of distal two-third gastric cancers had no. 6 metastasis were compatible with their results. However, after subdividing the no. 6 station into three substations,

\begin{tabular}{lllll}
\hline & $\begin{array}{l}\text { Any no. } 6^{\text {a }} \\
N(\%)\end{array}$ & $\begin{array}{l}\text { No. 6a } \\
N(\%)\end{array}$ & $\begin{array}{l}\text { No. 6v } \\
N(\%)\end{array}$ & $\begin{array}{l}\text { No. 6i } \\
N(\%)\end{array}$ \\
\hline $\mathrm{U}$ & & & & \\
$\mathrm{T} 1(N=24)$ & 0 & 0 & 0 & 0 \\
$>\mathrm{T} 2(N=24)$ & 0 & 0 & 0 & 0 \\
$\mathrm{M}$ & & & & 0 \\
$\mathrm{~T} 1(N=117)$ & $3(2.6)$ & $3(2.6)$ & 0 & $1(2.1)$ \\
$>\mathrm{T} 2(N=47)$ & $4(8.5)$ & $3(6.4)$ & $1(2.1)$ & $2(2.1)$ \\
$\mathrm{L}$ & & & & $8(19.5)^{*}$ \\
$\mathrm{~T} 1(N=95)$ & $5(5.3)$ & $3(3.2)$ & $2(2.1)$ & $11(3.2)$ \\
$>\mathrm{T} 2(N=41)$ & $19(45.3)^{*}$ & $10(24.4)^{*}$ & $12(29.3)^{*}$ & $15(4.3)$ \\
$\mathrm{T}$ Total $(N=348)$ & $31(8.9)$ & $19(5.5)$ & &
\end{tabular}

* $P<0.01$ as compared with $\mathrm{M}$

a The number does not add up because of metastases at plural substations 

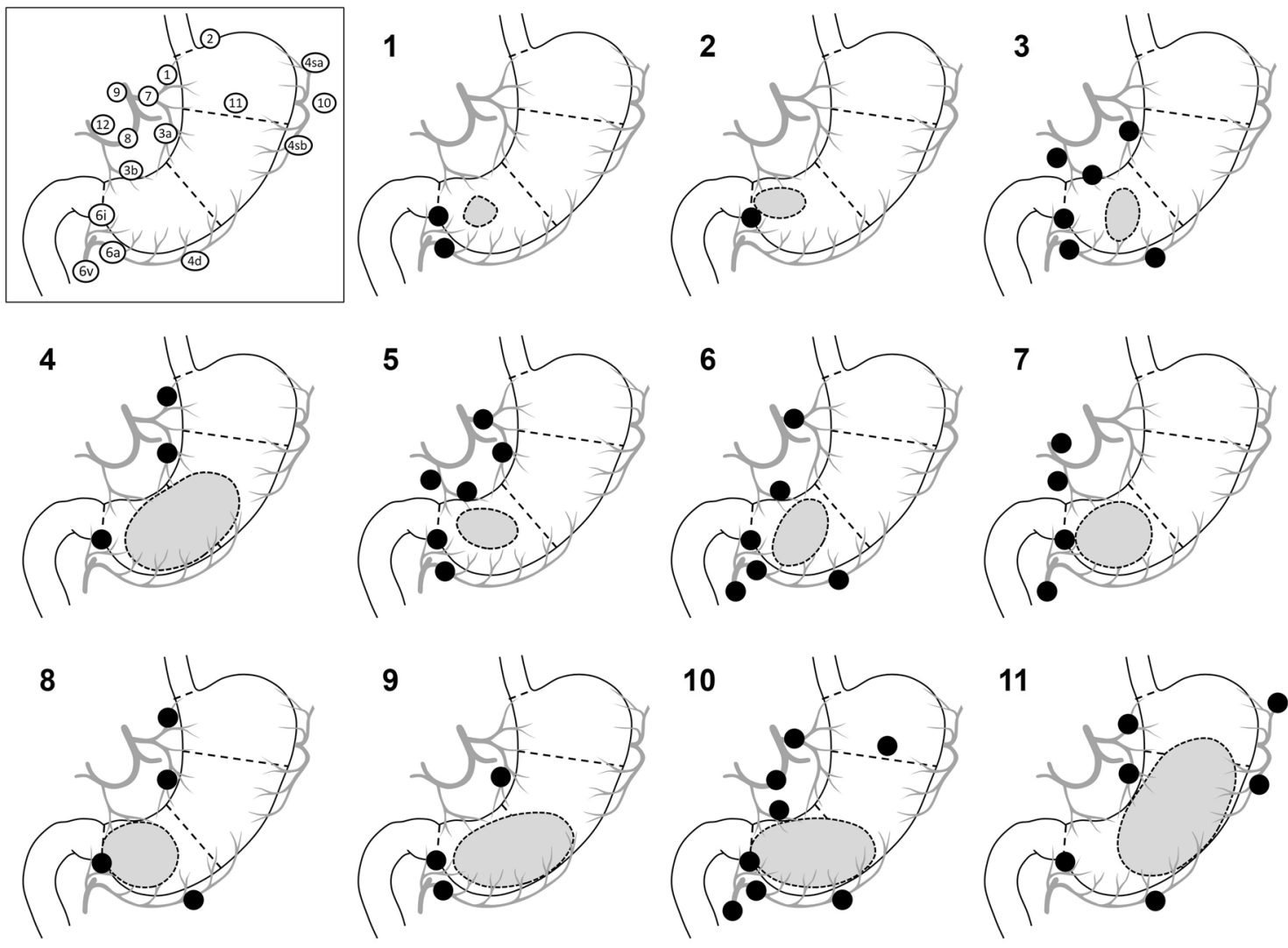

\begin{tabular}{lccccccccccc}
\hline Case no. & 1 & 2 & 3 & 4 & 5 & 6 & 7 & 8 & 9 & 10 & 11 \\
\hline Location & $\mathrm{L}$ & $\mathrm{L}$ & $\mathrm{L}$ & $\mathrm{L}$ & $\mathrm{L}$ & $\mathrm{L}$ & $\mathrm{L}$ & $\mathrm{L}$ & $\mathrm{L}$ & $\mathrm{L}$ & $\mathrm{M}$ \\
Depth of tumor invasion & $\mathrm{1b}$ & $1 \mathrm{~b}$ & 3 & 3 & $4 \mathrm{a}$ & $4 \mathrm{a}$ & $4 \mathrm{a}$ & $4 \mathrm{a}$ & $4 \mathrm{a}$ & $4 \mathrm{a}$ & $4 \mathrm{a}$ \\
Macroscopic type & $0-\mathrm{Ilc}$ & $0-\mathrm{Ilc}$ & 3 & 3 & 2 & 3 & 2 & 3 & 2 & 3 & 4 \\
Diameter $(\mathrm{mm})$ & 18 & 32 & 36 & 101 & 40 & 53 & 62 & 65 & 95 & 97 & 115 \\
Distance* $(\mathrm{mm})$ & 20 & 0 & 35 & 12 & 14 & 15 & 1 & 0 & 22 & 0 & 44 \\
\hline
\end{tabular}

Fig. 2 Characteristics of 11 gastric cancers with nodal metastasis along the infrapyloric artery. The gray oval in each figure roughly indicates the location and diameter of the tumor. Black circles

metastasis to the no. 6i LNs was observed in only 11 tumors. The majority of these tumors, including two early tumors, were located in $\mathrm{L}$, and the distance from the distal tumor border to the pyloric ring was no more than $44 \mathrm{~mm}$. The no. 6i metastasis rate of early lower-third tumors was $2.1 \%$, and the rate reached $19.5 \%$ in advanced tumors. These results are consistent with the anatomy of the IPA, which supplies the pyloric antrum for a length of $3-5 \mathrm{~cm}$ $[4,5]$, and indicate that lymphadenectomy along the IPA is an important part in no. $6 \mathrm{LN}$ dissection for treating gastric cancer invading the antrum. Further studies using dye imaging [11] would visualize the lymphatic stream in the infrapyloric region.

Clinically, T1N0 gastric cancer whose distal border is at least $4 \mathrm{~cm}$ proximal to the pylorus is a good indication for indicate metastasis-positive lymph node stations, which were depicted in the upper left panel. Asterisk indicates the distance from the distal tumor border to the pyloric ring

PPG [12]. This operation has an advantage over conventional distal gastrectomy, with a lower incidence of dumping syndrome, bile reflux gastritis, and nutritional deficit [13-16]. These advantages are attributable to preservation of the pyloric branch of the vagus nerve and the nutrient vessels supplying the antrum [17-20]. Since the rate of metastasis to nodes along the right gastric artery (no. 5 LNs) from early middle-third gastric cancer is known to be extremely rare [18, 21], omission of the suprapyloric LN dissection in PPG has been agreed upon by surgeons [12]. In contrast, the infrapyloric LN dissection has involved skeletonization of the IPA and the vein [20] while meticulously removing the soft tissue since no data about metastatic frequency at LNs along the vessels were available [19]. In the present study, there was no early 
middle-third gastric cancer patients with no. 6i metastasis, implying that lymphadenectomy along the IPA may be dispensable in PPG. Our results also showed that the incidence of no. $6 \mathrm{v}$ metastasis is as low as that of no. $6 \mathrm{i}$ in early middle-third gastric cancer. The therapeutic benefit of dissection of the no. 6 LNs in these cases is not negligible since the metastasis rate reached $2.6 \%$; the value is consistent with a report by Kong et al. [18]. However, considering that all metastases were localized in the no. $6 \mathrm{a}$ substation, lymphadenectomy along the RGEV below the level of the root of the RGEA may not be so essential when performing PPG.

In conclusion, in gastric cancer invading the antrum, lymphadenectomy along the IPA is important in radical surgery since metastasis to no. 6i LNs was frequent. In contrast, metastasis was not observed in early middle-third gastric cancer, suggesting that lymphadenectomy along the IPA may be dispensable when performing PPG. Further prospective studies are required to validate our hypothesis.

Acknowledgments We thank all the young surgeons at Toranomon Hospital for their assistance in manipulating surgical specimens.

\section{Compliance with ethical standards}

Funding This work was supported in part by the National Cancer Center Research and Development Funds (26-A-4) from the Ministry of Health, Labor, and Welfare of Japan.

Conflict of interest The authors declare that they have no conflict of interest.

Ethical approval and patient consent All patients gave their informed consent before the procedures were performed, and the study protocol was approved by the Ethics Committee of Toranomon Hospital (no. 1164).

\section{References}

1. Pólya E, von Navratil D. Untersuchung über die Lymphbahnen des Wurmfortsatzes und des Magens. Zeitschr für klin Chir. 1903;69:421-56 (German).

2. Sasako M, McCulloch P, Kinoshita T, Maruyama K. New method to evaluate the therapeutic value of lymph node dissection for gastric cancer. Br J Surg. 1995;82:346-51.

3. Association Japanese Gastric Cancer. Japanese classification of gastric carcinoma: 3rd English edition. Gastric Cancer. 2011;14:101-12.

4. Liberski SM, Koch KL, Atnip RG, Stern RM. Ischemic gastroparesis: resolution after revascularization. Gastroenterology. 1990;99:252-7.

5. Wilkie D. Blood supply of the duodenum. Surg Gynecol Obstet. 1911;3:399-405.
6. Haruta S, Shinohara H, Ueno M, Udagawa H, Sakai Y, Uyama I. Anatomical considerations of the infrapyloric artery and its associated lymph nodes during laparoscopic gastric cancer surgery. Gastric Cancer. 2015;18:876-80.

7. UICC International Union Against Cancer. TNM classification of malignant tumours. 7th ed. New York: Wiley; 2009. p. 73-7.

8. Shinohara H, Kurahashi Y, Kanaya S, Haruta S, Ueno M, Udagawa $\mathrm{H}$, et al. Topographic anatomy and laparoscopic technique for dissection of no. 6 infrapyloric lymph nodes in gastric surgery. Gastric Cancer. 2013;16:615-20.

9. Shinohara H, Haruta S, Ohkura Y, Udagawa H, Sakai Y. Tracing dissectable layers of mesenteries overcomes embryological restrictions when performing infrapyloric lymphadenectomy in laparoscopic gastric cancer surgery. J Am Coll Surg. 2015;220:e81-7.

10. Maruyama K, Gunvēn P, Okabayashi K, Sasako M, Kinoshita T. Lymph node metastases of gastric cancer. General pattern in 1931 patients. Ann Surg. 1931;1989(210):596-602.

11. Nishigori N, Koyama F, Nakagawa T, Nakamura S, Ueda T, Inoue $\mathrm{T}$, et al. Visualization of lymph/blood flow in laparoscopic colorectal cancer surgery by ICG Fluorescence Imaging (LapIGFI). Ann Surg Oncol. 2016;23:S266-74.

12. Association Japanese Gastric Cancer. Japanese gastric cancer treatment guidelines 2010 (ver. 3). Gastric Cancer. 2011;14:113-23.

13. Tomita R, Fujisaki S, Tanjoh K. Pathophysiological studies on the relationship between postgastrectomy syndrome and gastric emptying function at 5 years after pylorus-preserving distal gastrectomy for early gastric cancer. World J Surg. 2003;27:725-33.

14. Shibata C, Shiiba KI, Funayama Y, Ishii S, Fukushima K, Mizoi $\mathrm{T}$, et al. Outcomes after pylorus-preserving gastrectomy for early gastric cancer: a prospective multicenter trial. World J Surg. 2004;28:857-61.

15. Nunobe S, Sasako M, Saka M, Fukagawa T, Katai H, Sano T. Symptom evaluation of long-term postoperative outcomes after pylorus-preserving gastrectomy for early gastric cancer. Gastric Cancer. 2007;10:167-72.

16. Park DJ, Lee HJ, Jung HC, Kim WH, Lee KU, Yang HK. Clinical outcome of pylorus-preserving gastrectomy in gastric cancer in comparison with conventional distal gastrectomy with Billroth I anastomosis. World J Surg. 2008;32:1029-36.

17. Shinohara H, Sonoda T, Niki M, Nomura E, Nishiguchi K, Tanigawa N. Laparoscopically-assisted pylorus-preserving gastrectomy with preservation of the vagus nerve. Eur J Surg. 2002;168:55-8.

18. Kong SH, Kim JW, Lee HJ, Kim WH, Lee KU, Yang HK. The safety of the dissection of lymph node stations 5 and 6 in pyloruspreserving gastrectomy. Ann Surg Oncol. 2009;16:3252-8.

19. Hiki N, Kaminishi M. Pylorus-preserving gastrectomy in gastric cancer surgery: open and laparoscopic approaches. Langenbecks Arch Surg. 2005;390:442-7.

20. Nishizawa N, Yamashita K, Shinohara H, Sakuramoto S, Hosoda $\mathrm{K}$, Watanabe M. Anatomical knowledge for the infra-pyloric vein preservation during the laparoscopy-assisted pylorus-preserving gastrectomy. Dig Surg. 2016;33:363-70. doi:10.1159/000445069.

21. Kim BH, Hong SW, Kim JW, Choi SH, Yoon SO. Oncologic safety of pylorus-preserving gastrectomy in the aspect of metastasis in lymph nodes at stations 5 and 6. Ann Surg Oncol. 2014;21:533-8. 